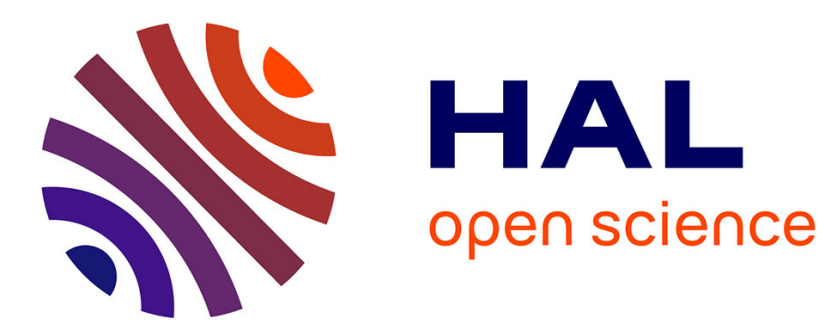

\title{
Superconductivity exponents in two- and three-dimensional percolation
}

H. j. Herrmann, Bernard Derrida, J. Vannimenus

\section{To cite this version:}

H. j. Herrmann, Bernard Derrida, J. Vannimenus. Superconductivity exponents in two- and threedimensional percolation. Physical Review B, 1984, 30 (7), pp.4080-4082. 10.1103/PhysRevB.30.4080 . hal-03285926

\section{HAL Id: hal-03285926 \\ https://hal.science/hal-03285926}

Submitted on 21 Jul 2021

HAL is a multi-disciplinary open access archive for the deposit and dissemination of scientific research documents, whether they are published or not. The documents may come from teaching and research institutions in France or abroad, or from public or private research centers.
L'archive ouverte pluridisciplinaire HAL, est destinée au dépôt et à la diffusion de documents scientifiques de niveau recherche, publiés ou non, émanant des établissements d'enseignement et de recherche français ou étrangers, des laboratoires publics ou privés. 


\title{
Superconductivity exponents in two- and three-dimensional percolation
}

\author{
H. J. Herrmann and B. Derrida \\ Service de Physique Théorique, Centre d'Etudes Nucléaires de Saclay, \\ F-91191 Gif-sur-Yvette Cedex, France \\ J. Vannimenus \\ Laboratoire de Physique des Solides, Ecole Normale Supérieure, \\ 24 rue Lhomond, F-75231 Paris Cedex 05, France
}

(Received 9 February 1984; revised manuscript received 22 May 1984)

\begin{abstract}
The first transfer-matrix calculation of the superconductivity exponent $s$ of a random mixture of normal and superconducting elements is presented: The exponent $s$ is defined through the divergence of the conductivity $\Sigma$ as the critical fraction $p_{c}$ of superconducting elements is approached: $\Sigma \sim\left(p-p_{c}\right)^{-s}$. We obtain very accurate values for the exponents which disagree with the Alexander-Orbach conjecture as well as other conjectures. Our results are $s / \nu=0.977 \pm 0.010$ in two dimensions and $s / \nu=0.85 \pm 0.04$ in three dimensions.
\end{abstract}

The conductivity $\Sigma$ of a random mixture of superconducting (fraction $p$ ) and normal-conducting (fraction $1-p$ ) elements diverges for $p \rightarrow p_{c}^{-}$as

$$
\Sigma \sim\left(p_{c}-p\right)^{-s}
$$

where $p_{c}$ is the percolation threshold. We will only treat bond percolation in this Rapid Communication. The exponent $s$ plays an important role in the transport properties of random systems. It has been of theoretical ${ }^{1-7}$ and experimental $^{8-11}$ interest, and some conjectures about its value have been proposed. ${ }^{7,12,13}$ It appears, in particular, in the critical behavior of the dielectric constant, 5,10 in the absorption coefficient ${ }^{11}$ of random metal-insulator composites, in the conduction of binary metallic mixtures, ${ }^{1,6}$ as well as in the viscosity of a gel. ${ }^{4}$

In two dimensions, the exponent $s$ has been shown by an exact duality ${ }^{1}$ to be equal to the conductivity exponent $t$ of a mixture of conducting and insulating elements above $p_{c}$. On the contrary, in three dimensions, no exact relation between $s$ and $t$ is known. The conductivity exponent $t$ has been numerically calculated by different methods, such as series expansions, ${ }^{14}$ relaxation methods, ${ }^{15}$ diffusion, ${ }^{16}$ and transfer matrices. ${ }^{17,18}$ Numerical calculations of $s$, however, are difficult. We will describe, in this paper, a new method based on a transfer-matrix algorithm which allows a very precise determination of $s$, and we will present numerical results for dimensions $d=2$ and $d=3$. In $d=2$, we can compare our results to the existing numerical determinations of $t$.

Let us first describe our method. We construct, in $d=2$, a $n \times L$ strip of a square lattice as shown in Fig. 1, and in

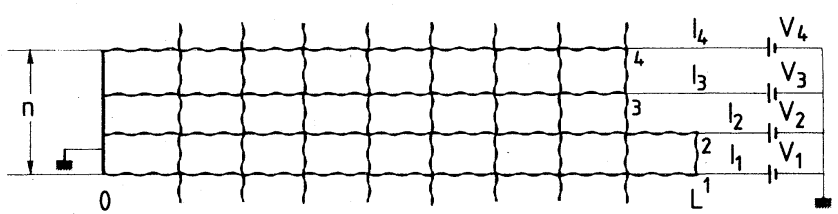

FIG. 1. Two-dimensional strip of conductors (some are normal and some superconducting). The strip has width $n=4$ and periodic boundary conditions in vertical direction. At column 0 the voltage is 0 and at column $L$, which is just being constructed, the $i$ th row has a current $I_{i}$ coming from a voltage $V_{i}$. $d=3$, a $n \times n \times L$ bar of a cubic lattice going to $L \rightarrow \infty$ by adding bond after bond with a resistance randomly chosen 0 with probability $p_{c}$, and $r$ with probability $1-p_{c}$. In the $d-1$ vertical directions, we impose periodic boundary conditions. This is possible in this case, because the current flows along the strip. In the case of normal conductivity, such a geometry is not possible because the current would be zero and so one has to consider a current flowing across the strip, i.e., the two boundaries having different potential. ${ }^{17}$ In the first vertical plane, we impose the voltage $V=0$ and look for the voltages $V_{i}$ which should be put on each of the $N=n^{d-1}$ end points in the $L$ th vertical plane in order that they carry a current $I_{i}$ (see Fig. 1). They are given through the resistivity matrix $B_{i j}$ by

$$
V_{i}=\sum_{j=1}^{N} B_{i j} I_{j}, \quad i=1 \text { to } N .
$$

For each bond that one adds, $B_{i j}$ has to be updated. The updated matrix $B_{i j}^{\prime}$ is

$$
B_{i j}^{\prime}=B_{i j}+r \delta_{i \alpha} \delta_{j \alpha}
$$

if one adds a horizontal bond at line $\alpha$, and is

$$
B_{i j}^{\prime}=B_{i j}-\frac{\left(B_{i \alpha}-B_{i \beta}\right)\left(B_{\alpha j}-B_{\beta j}\right)}{r+\left(B_{\alpha \alpha}+B_{\beta \beta}-B_{\alpha \beta}-B_{\beta \alpha}\right)}
$$

if one adds a vertical bond between lines $\alpha$ and $\beta$. We note that the generalization to the case of anisotropy is straightforward. The conductivity $\Sigma$ (or resistivity $R$ ) of a strip defined by $\Sigma^{-1}=R=B_{i i} / L$ is well defined and independent of the end point $i$, in the limit $L \rightarrow \infty$. Also in this limit, it is not necessary to average over several samples. Thus, we calculate up to a value of $L$ as large as possible to diminish the statistical error. These statistical errors were estimated by dividing the strip into roughly 50 parts. The calculation is performed for different widths $n$, at the percolation threshold $p_{c}$, yielding conductivities $\Sigma_{n}$, and one then extracts the critical exponents through finite-size scaling:

$$
\Sigma_{n} \sim n^{s / \nu}\left(1+b n^{-\omega}+\cdots\right) \text {. }
$$

Knowing the correlation length exponent $\nu$, one thus obtains from the large- $n$ behavior, the exponent $s$.

In two dimensions, we know $p_{c}=\frac{1}{2}, \nu=\frac{4}{3}$, and $s=t$. For 
$t$, Alexander and Orbach ${ }^{13}$ proposed the conjecture $t=\frac{91}{72}$. To directly test this conjecture we show, in Fig. 2, a log-log plot of $n^{91 / 96} / \Sigma_{n}$ against $n . L$ is taken up to $10^{7}$. If the conjecture was correct, the curve should become horizontal for large $n$, which in view of Fig. 2, seems very unlikely. A careful analysis that takes into account the correction to scaling of Eq. (5) and the statistical errors, similar to the analysis that we will describe for three dimensions, yields

$$
\frac{s}{\nu}=0.977 \pm 0.010,
$$

clearly not including the value $s / \nu \simeq 0.948$ predicted by the conjecture. Our value is in good agreement with less precise determinations of $t^{16,17,19}$ and confirms a recent, very accurate calculation by Zabolitzky: ${ }^{18} t / \nu=0.973 \pm 0.005$. The work of Ref. 18 was based on a transfer-matrix algorithm for the conductivity problem. Our result, Eq. (6), comparable in precision, was obtained with much less effort than that of Ref. 18 (10 times smaller widths, 100 times less computing time): we believe that one of the reasons is that for the superconductivity problem we could use, contrary to Ref. 18, periodic boundary conditions in the vertical direction. With these conditions, finite-size scaling already works well with moderate widths (see, e.g., Ref. 20). In the calculations performed by Zabolitzky, ${ }^{18}$ the fixed-boundary conditions mix finite-size effects with surface effects and therefore it is necessary to use wider strips to compensate for these surface effects. On the contrary, in our case, the

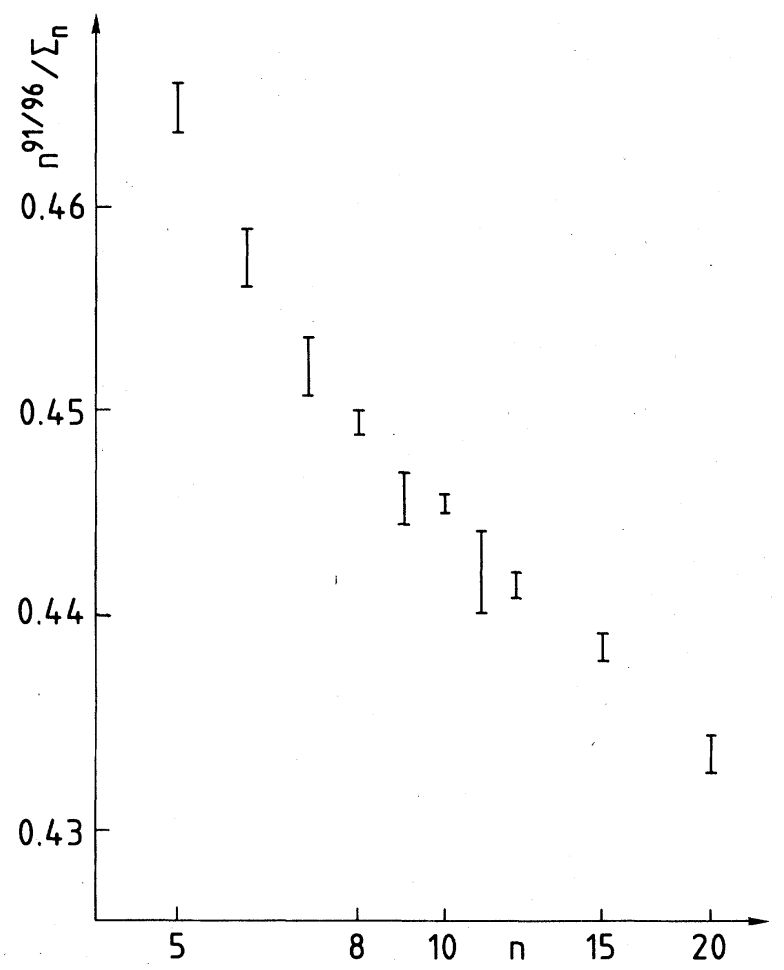

FIG. 2. Log-log plot of $n^{91 / 96} / \Sigma_{n}$ against the strip width $n$ in two dimensions. Error bars arise from the statistical errors $\left(L=10^{7}\right.$ for $n=8,10,12,15,20$ and $L=10^{6}$ for $n=5,7,9,11$ ). If the Alexander-Orbach conjecture were correct, the curve would be horizontal for large $n$. periodic boundary conditions generate only finite-size effects. ${ }^{21}$

In three dimensions, since $p_{c}$ is not known exactly, we use $p_{c}=0.2492 .^{22}$ Kertész $^{7}$ has proposed for $s$ the conjecture $s=\nu-\beta / 2$ for all $d$, agreeing for $d=2$ with Alexander and Orbach. For $d=3$, the conjecture yields $s / \nu=0.744 \pm 0.017 .^{23}$ Thus, we plot in the same spirit as above $n^{0.744} / \Sigma_{n}$ against $n$ in Fig. 3, with $L$ being of the order of $10^{6}$. Again the curve should become horizontal if the conjecture is correct. As with Fig. 2, already an extrapolation by eye does not seem to allow for an asymptotic slope for large $n$ within the two dashed lines shown in Fig. 3 that correspond to the conjecture of Kertész. Looking at Fig. 3, one already sees that the points from $n=5-10$ lie on one straight line of slope 0.89 , but the curvature ensures that this is an upper bound.

Let us briefly discuss our correction to scaling analysis for the data of Fig. 3, the same analysis that in two dimensions led to Eq. (6). For a given correction to scaling exponent $\omega$, we fit a curve of the form of Eq. (5) to the data, and the best values of $s / \nu$ so obtained are shown in Fig. 4. This is not only done for the mean values of the data but, also for the upper or lower limits of the error bars. The $s / \nu$ obtained in these different ways all lie within the dashed re-

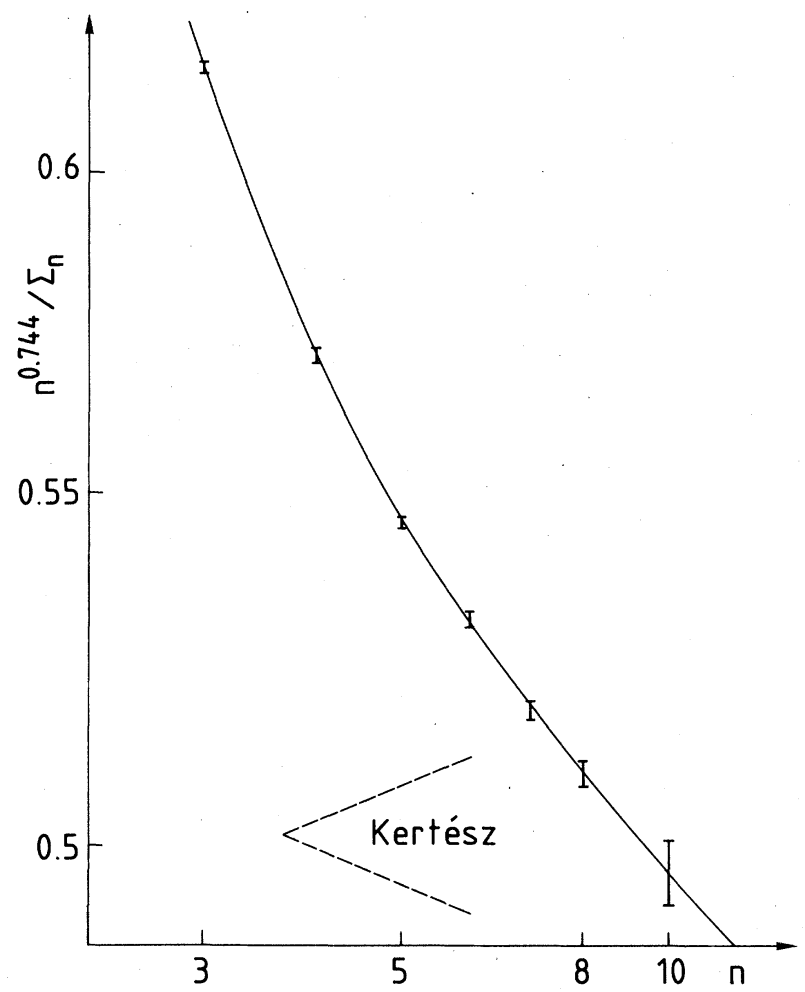

FIG. 3. Log-log plot of $n^{0.744} / \Sigma_{n}$ against the bar width $n$ in three dimensions. The error bars correspond to $L=10^{6}$ for $n \leqslant 7$, $L=7 \times 10^{5}$ for $n=8$, and $L=3 \times 10^{5}$ for $n=10$. The conjecture of Kertész (Ref. 7) would be correct if the asymptotic slope for large $n$ lay between the two dashed slopes. The line drawn through the points is one of the possible fits through the points including corrections to scaling and corresponds to $\Sigma_{n}=n^{-0.847}(0.626$ $\left.+0.912 n^{-2.4}\right)$. The total computer time required for obtaining this data was of the order of $30 \mathrm{~h}$ on a CRAY 1 . 
gion of Fig. 4. The $\chi^{2}$ test limits the interval of $\omega$ to be between $2.2<\omega<2.8$ as shown in Fig. 4. This leads to

$$
\frac{s}{\nu}=0.85 \pm 0.04
$$

where the error bars include, as explained above, not only the uncertainty in $\omega$ but also the statistical error. If one noticeably deviates from the given $\omega$ range, $\chi^{2}$ quickly increases and the amplitude of the correction term becomes unreasonably large. We note that in two dimensions, the range of $\omega$ given by the $\chi^{2}$ test is $1.8<\omega<2.7$.

Using $\nu \approx 0.88$, given in Ref. 23, we get $s=0.75 \pm 0.04$. This value excludes the conjecture of Kertész ${ }^{7}$ but agrees, rather well, with real-space renormalization calculations $s=0.77$ (Ref. 3) and experimental measurements $s=0.73$ \pm 0.07 . $^{11}$

In conclusion, we found, with a new method based on the transfer-matrix technique, very precise values for the superconductivity exponents $s$ in two and three dimensions. Our ranges of values clearly do not include the values conjectured by Alexander and Orbach, and by Kertész, but they are in agreement with experiments ${ }^{11}$ and with a recent cal-

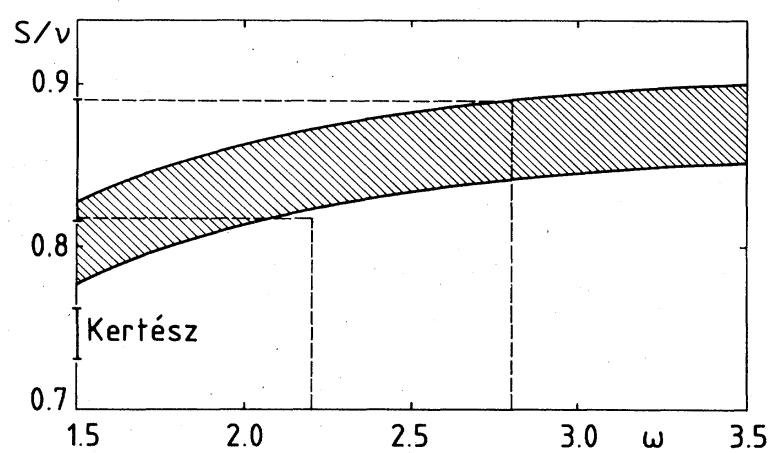

FIG. 4. Corrections to scaling analysis of the data shown in Fig. 3. The extrapolated values, as well as the conjecture by Kertész, are marked by black segments. The dashed lines limit the range of values of $\omega$ obtained from the $\chi^{2}$ test.

culation of Zabolitzky. ${ }^{18}$ Our results apply, in particular, to a problem recently formulated in Ref. 24 .

We acknowledge the Centre National de la Recherche Scientifique for giving us computer time on the CRAY 1.
1J. P. Straley, Phys. Rev. B 15, 5733 (1977).

2J. P. Straley, in Electrical Transport and Optical Properties of Inhomogeneous Media (Ohio State University, 1977), AIP Conf. Proc. No. 40, edited by J. C. Garland and D. B. Tanner (AIP, New York, 1978), p. 118, and references therein.

3J. Bernasconi, Phys. Rev. B 18, 2185 (1978).

${ }^{4}$ P. G. de Gennes, J. Phys. (Paris) Lett. 40, L197 (1979).

${ }^{5}$ D. Wilkinson, J. S. Langer, and P. N. Sen, Phys. Rev. B 28, 1081 (1983).

${ }^{6}$ R. Fogelholm and G. Grimvall, J. Phys. C 16, 1077 (1983); S. A. Solla and N. W. Ashcroft (unpublished).

${ }^{7}$ J. Kertész, J. Phys. A 16, L471 (1983).

${ }^{8}$ C. J. Lobb, M. Tinkham, and W. J. Skocpol, Solid State Commun. 27, 1273 (1978).

${ }^{9}$ Y. M. Gerbshtein, T. V. Smirnova, E. I. Terukov, and F. A. Chudnovskii, Fiz. Tverd. Tela (Leningrad) 18, 503 (1976) [Sov. Phys. Solid State 18, 290 (1976)].

10D. M. Girannan, J. C. Garland, and D. B. Tanner, Phys. Rev. Lett. 46, 375 (1981).

${ }^{11}$ D. R. Bowman and D. Stroud, Phys. Rev. Lett. 52, 299 (1984).

${ }^{12}$ R. Rammal and G. Toulouse, J. Phys. (Paris) Lett. 44, L13 (1983).
${ }^{13}$ S. Alexander and R. Orbach, J. Phys. (Paris) Lett. 43, L625 (1982).

${ }^{14}$ R. Fish and A. B. Harris, Phys. Rev. B 18, 416 (1978).

${ }^{15}$ S. Kirkpatrick, Rev. Mod. Phys. 45, 574 (1973).

${ }_{16}$ R. B. Pandey and D. Stauffer, Phys. Rev. Lett. 51, 527 (1983).

${ }^{17}$ B. Derrida and J. Vannimenus, J. Phys. A 15, L557 (1982); B. Derrida, D. Stauffer, H. J. Herrmann, and J. Vannimenus, J. Phys. (Paris) Lett. 44, L701 (1983); B. Derrida, J. G. Zabolitzky, J. Vannimenus, and D. Stauffer, J. Stat. Phys. (to be published).

18J. G. Zabolitzky, Phys. Rev. B 30, 4076 (1984) (preceding paper).

${ }^{19}$ C. J. Lobb and D. J. Frank, J. Phys. C 12, L827 (1979); P. S. Li and W. Strieder, J. Phys. C 15, L1235 (1982); M. Sahimi, B. Hughes, L. E. Scriven, and H. T. Davis, J. Phys. C 16, L521 (1983).

${ }^{20}$ B. Derrida and L. de Seze, J. Phys. (Paris) 43, 475 (1982).

${ }^{21}$ E. Brézin, in Fifth International Conference on Collective Phenomena, edited by J. Lebowitz, Annals of the New York Academy of Sciences, Vol. 410 (New York Academy of Sciences, New York, 1983), p. 339.

${ }^{22}$ S. Wilke, Phys. Lett. A (to be published).

${ }^{23}$ D. W. Heermann and D. Stauffer, Z. Phys. B 44, 339 (1981).

${ }^{24}$ A. Coniglio and H. E. Stanley, Phys. Rev. Lett. 52, 1068 (1984). 